\title{
Biological vulnerability to depression: linked structural and functional brain network findings
}

N. L. Nixon, P. F. Liddle, E. Nixon, G. Worwood, M. Liotti and L. Palaniyappan

\section{Background}

Patients in recovery following episodes of major depressive disorder (MDD) remain highly vulnerable to future recurrence. Although psychological determinants of this risk are well established, little is known about associated biological mechanisms. Recent work has implicated the default mode network (DMN) in this vulnerability but specific hypotheses remain untested within the high risk, recovered state of $\mathrm{MDD}$

\section{Aims}

To test the hypothesis that there is excessive DMN functional connectivity during task performance within recovered-state MDD and to test for connected DMN cortical gyrification abnormalities.

\section{Method}

A multimodal structural and functional magnetic resonance imaging (fMRI) study, including task-based functional connectivity and cortical folding analysis, comparing 20 recoveredstate patients with MDD with 20 matched healthy controls.

\section{Results}

The MDD group showed significant task-based DMN hyperconnectivity, associated with hypogyrification of key DMN regions (bilateral precuneus).

\section{Conclusions}

This is the first evidence of connected structural and functional DMN abnormalities in recovered-state MDD, supporting recent hypotheses on biological-level vulnerability.

\section{Declaration of interest}

N.L.N. received funding from the Institute of Mental Health, Nottingham, which enabled him to carry out this research project, and has received financial assistance to attend academic meetings from Janssen-Cilag, AstraZeneca, Servier and Shire. He has also taken part in advisory panels for Janssen-Cilag and Servier. P.F.L. has received honoraria for academic presentations from GlaxoSmithKline, AstraZeneca, Janssen-Cilag, Bristol-Myers Squibb, Eli Lilly and Johnson \& Johnson Pharmaceuticals. He has also taken part in advisory panels for Eli Lilly, Pfizer and GlaxosmithKline. G.W. has received honoraria for academic presentations from JanssenCilag. M.L. received a grant from the National Alliance for Research on Schizophrenia and Depression (NARSAD). L.P. has received a travel fellowship from the International Bipolar Disorder Society sponsored by Eli Lilly in 2010.
Patients who have recovered from episodes of major depressive disorder (MDD) remain highly vulnerable to future recurrence and clinical studies put this risk of further MDD episodes at up to $80 \%,{ }^{1}$ in contrast with the general population lifetime risk of $6.7 \%$ (95\% CI 4.2-10.1). ${ }^{2}$ Although psychological determinants of this risk are well established, ${ }^{3,4}$ relatively little is known about associated biological mechanisms. Functional magnetic resonance imaging (fMRI) studies have provided some early data on the presence of functional brain abnormalities in recovered-state patients with MDD when performing executive tasks. ${ }^{5,6}$ However, there remains no published evidence on brain network functional connectivity or related structure in recovered MDD despite credible recent hypotheses of their importance. The clearest theory concerns the default mode network (DMN); a brain network in which increased levels of activity have well-evidenced associations with internally focused appraisal ${ }^{7}$ and that has therefore been considered a potential neural substrate for the ruminative, introspective cognitive patterns of MDD. ${ }^{8-10}$ The DMN is further implicated through convergent findings of increased resting-state functional connectivity between specific DMN regions (namely the dorsomedial prefrontal cortex and precuneus) in both depressed-state $\mathrm{MDD}^{11}$ and never-depressed, first-degree relatives of patients with recurrent MDD. ${ }^{9}$ This has led the authors of a recent review to hypothesise that DMN hyperconnectivity continues within recovered-state MDD, persisting into task-based activity where it acts as a substrate for overly internal processing, interfering with the recruitment of more effective networks and ultimately creating biological-level vulnerability to depression. ${ }^{10}$ Recent evidence linking functional connectivity and gyrification of the cortical surface ${ }^{12}$ means we might additionally predict that abnormal DMN functional connectivity is associated with abnormal gyrification in key DMN regions. In light of this literature, our aims were first to test the hypothesis that there is excessive task-based DMN functional connectivity within recovered-state MDD; and second to test the hypothesis that there are connected abnormalities in cortical gyrification within anatomical regions of the DMN.

\section{Method}

\section{Recruitment and clinical assessment}

The recruitment and assessment of this population has been previously reported in detail, ${ }^{5}$ but is summarised here. Patients (the MDD group) were recruited through physician referral from general adult psychiatric clinics in Nottingham, UK. The control group were mainly recruited through posters displayed at a general hospital and a community surgery in Nottingham. Following complete description of the study, written, informed, capacitous consent was obtained from all participants. The study was approved by the local research ethics committee.

All potential recruits underwent an initial psychiatric assessment by senior clinical psychiatrists (N.L.N., G.W., P.F.L.), including a detailed history of personal and family psychiatric/ medical disorder, substance misuse and medication history; followed by the Structured Clinical Interview for DSM-IV Axis I Disorders (SCID-CV); ${ }^{13}$ two measures of depressive symptoms, the 17-item Hamilton Rating Scale for Depression (HRSD) ${ }^{14}$ 
and the Beck Depression Inventory - II (BDI-II); ${ }^{15}$ two measures of personality, the Short Eysenck Personality Questionnairerevised (EPQ-R $)^{16}$ and the Personality Disorders Questionnaireversion 4 (PDQ-4+); ${ }^{17}$ a measure of IQ, the Ammons Quick Test (Quick) $;^{18}$ a measure of cognitive function, the Folstein MiniMental State Examination (MMSE) $;{ }^{19}$ a handedness questionnaire (RIC); $;^{20}$ and an fMRI safety questionnaire.

A clinical consensus meeting followed initial screening to determine inclusion/exclusion. Minimum inclusion for the MDD group required at least two previous episodes of DSM-IV major depression, ${ }^{21}$ diagnosed through SCID-CV, now in a recovered-state (with normalised function for at least 3 months and HRSD score $<8$ ). Exclusion criteria were comorbid Axis I psychiatric disorder (with particular focus on misdiagnosed bipolar II disorder or anxiety disorder); personality disorder; drug or alcohol disorder; untreated medical disorder; any previous or current central nervous system (CNS) disease; or fMRI safety issues. In addition to the above criteria, potential controls were excluded if there was evidence of any current or past psychiatric disorder. Patients were followed up over 1 year for evidence of recurrence, determined through use of the SCID-CV at 4-month intervals combined with clinical data from case notes and treating clinicians.

Statistical testing of clinical, demographic and behavioural data used SPSS 16.0 on Windows and applied the significance criterion $P<0.05$, with equal variances not assumed. All $t$-tests were 2-tailed.

\section{Image acquisition}

Blood oxygen level-dependent (BOLD) data acquisition: gradient echo-echo planar images were acquired using a Philips 3T system during two separate $10 \mathrm{~min}$ sessions of a Go/No-Go (GNG) paradigm. Following $T_{2^{*}}$ image stabilisation, 252 volumes of 36 contiguous descending slices were collected (image matrix $64 \times 64$, voxel size $3 \mathrm{~mm} \times 3 \mathrm{~mm} \times 3 \mathrm{~mm}$, field of view $19.2 \mathrm{~cm}$, echo time $40 \mathrm{~ms}$ and repetition time $2300 \mathrm{~ms}$ ). All participants received standardised preparation aimed at minimising performance variance and anxiety (including scripted explanation, practice sessions of the task and habituation within the scanner).

The GNG paradigm has been previously reported in detail ${ }^{5}$ but is summarised here. Go (x) or No-Go (k) stimuli were presented on a projected screen for $267 \mathrm{~ms}$; interstimulus interval was jittered and pseudorandom between 3100 and $3700 \mathrm{~ms}$, so that each participant had either 159 or 160 stimuli per block of which 32 or 33 were No-Go. Participants were asked to respond as quickly as possible (by a single button press) when shown the letter ' $\mathrm{x}$ ' and to withhold response when shown the letter ' $\mathrm{k}$ '. The only explicit feedback used during task was the message 'too slow', shown for correct responses to ' $x$ ' that were delayed beyond an individually calculated time ceiling, derived from response times in the pre-fMRI practice sessions.

\section{BOLD data functional connectivity analysis}

First-level analysis

The PAR/REC format data from the 3T Philips system were converted to NIfTI format using the program dci2nii (www. mccauslandcenter.sc.edu/mricro/mricron/dcm2nii.html) based on MRIcron (www.mccauslandcenter.sc.edu/mricro/mricron). The converted images were then pre-processed using DPARSFA (www.restfmri.net) ${ }^{22}$ based on REST (www.restfmri.net) and SPM8 (www.fil.ion.ucl.ac.uk/spm). During pre-processing, images were reoriented, slice timed, spatially realigned, co-registered, segmented, normalised and smoothed to $8 \mathrm{~mm}$. The fMRI time series was then subject to linear detrending and temporal bandpass filtering $(0.01-0.08 \mathrm{~Hz})$ before regressing out motion parameters, global mean signal, white matter signal and cerebrospinal fluid signal (aimed at removing spurious fluctuations not involved in specific regional correlations). Following methodology validated in task-based functional connectivity analysis, ${ }^{23,24}$ transformed correlation coefficients ( $z$-scores) were calculated to generate seed-region based functional connectivity $\mathrm{z}(\mathrm{FCz})$ maps in DPARSFA, based on $8 \mathrm{~mm}$ radius seeds in the left and right precuneus (essential regional components of the DMN, with coordinates taken from key published literature ${ }^{11}$ ). Since the coordinates were initially published in Talairach space, a conversion was made from Talairach to Montreal Neurological Institute (MNI) space for the current analysis, using tal2mni. ${ }^{25}$ For each participant $\mathrm{FCz}$ maps were generated independently for the left and right DMN seeds, each containing the voxel-wise Pearson coefficients of the correlation between the time-series of BOLD variations in the seed region and the rest of the brain.

\section{Second-level analysis}

This comprised a main analysis, in which single-participant $\mathrm{FCz}$ maps were entered into $t$-tests, using SPM8, to assess whole-brain significance across-group and between-group (controls $v$. MDD group); a subsidiary analysis, assessing maintenance-phase medication effects, in which single-participant $\mathrm{FCz}$ maps were entered into one-factor analyses of variance (ANOVAs) with three levels (unmedicated patients, medicated patients, controls); and a region of interest (ROI) analysis to enable direct comparison of our findings with key literature. The ROI analysis used a small volume correction (SVC) within a $12 \mathrm{~mm}$ radius of bilateral dorsomedial prefrontal cortex regions identified by Sheline et al as uniquely important 'dorsal nexus' areas within depressed-state MDD exhibiting increased resting-state functional connectivity to several networks including the DMN. ${ }^{11}$ All between-group tests used age and session as covariates of no interest.

\section{Significance testing}

In keeping with Chumbley \& Friston, ${ }^{26}$ significance testing in our main and ROI analysis incorporates spatial extent, reporting clusters surviving correction at false discovery rate-corrected $P$ $\left(P_{\text {FDR-cor }}\right)<0.05$. Voxel-level statistics are additionally reported where appropriate at family-wise error-correct $P\left(P_{\text {FWE-cor }}\right)<0.05$ (for example in the case of extensive clusters crossing anatomically defined regions). Since cluster-level statistics are not provided in SPM8 ANOVA, this part of the subsidiary analysis reports voxel-level $P_{\text {FWE-cor }}<0.05$.

For ease of comparison with existing literature, all coordinates have been reported in Talairach space, following conversion from MNI space using the program mni2tal. ${ }^{24}$ Significant clusters have been illustrated with Xjview Version 8.11 (www.alivelearn.net/xjview).

\section{$T_{1}$ image gyrification analysis}

\section{Surface extraction}

Cortical surfaces were reconstructed using FreeSurfer version 5.1.0 on Mac OSX 10.5.8 (http://surfer.nmr.mgh.harvard.edu). Standard procedures were followed, as described by Dale et al. ${ }^{27}$ Pre-processing used Schaer's method, ${ }^{28}$ an extension of Zilles' gyrification index approach, ${ }^{29}$ to measure cortical folding patterns for each of the several thousands of vertices across the entire cortical surface. This automated method provides local gyrification indices (LGIs), numerical values assigned in a continuous fashion to each vertex of the reconstructed cortical surface. The LGI of a vertex corresponds to the ratio of the surface area of the folded pial contour ('buried' surface) to the outer contour of the cortex ('visible' surface) included within a sphere of $25 \mathrm{~mm}$ radius drawn around each vertex, in line with previous studies..$^{27,29,30}$ Thus the 
LGI value at each vertex reflects the amount of cortex buried in its immediate locality. Schaer's index captures both the spatial frequency and the amount of curvature of cortical folds in the locality of each vertex.

\section{Statistical analysis examining the spatial distribution} of group differences in gyrification

A vertex-wise whole brain analysis was performed to localise brain regions showing the most prominent gyrification differences between groups, assessing gyrification on a point-by-point basis across the entire brain. The vertex-wise LGI measurement for each participant was mapped on a common spherical coordinate system (fsaverage). A general linear model controlling for the effect of age and gender was used to compute differences in gyrification between the groups for the right and left hemispheric surfaces, in keeping with published literature. ${ }^{30,31}$ The FreeSurfer version 5.1.0 on Mac OSX 10.5.8 query design estimate contrast (QDEC) tool was used to generate between-group contrasts. To correct for multiple testing we used a permutation method with 10000 simulations and identified clusters that survived a type 1 error rate of $5 \%$ at a cluster inclusion threshold of $P=0.05$ (two-tailed). Reported results are corrected for age and gender (including intracranial volume (ICV) as a covariate in the model did not make any important or significant difference to these results).

To test for spatial overlap in the DMN nodes used in our functional connectivity analysis (right and left precuneus) and regions showing prominent gyrification defects, we used mri_vol2surf option in FreeSurfer to project these DMN nodes onto the reconstructed average cortical surface (fsaverage), alongside the clusters that emerged as significant in the vertex-wise whole-brain gyrification analysis.

\section{Results}

\section{Participants, clinical and behavioural data}

The demographic and clinical data have been previously reported in detail ${ }^{5}$ but are summarised here and in Table 1 . In total, 42 individuals (20 patients and 22 controls) met the minimum inclusion/exclusion criteria and hence were recruited. Complete $T_{2}{ }^{*}$ images sensitive to BOLD contrast were collected for 20 patients and 20 matched controls (the first 2 control participants were excluded because of technical difficulties with image acquisition).

Demographic data indicated that the control and MDD groups were well matched for age (range 24-63 years), gender, IQ and laterality. The MDD group showed high recurrence (mean of four episodes) but were in stable recovery at the time of data acquisition (mean 13 months).

Paired across-group $t$-tests $(n=40)$ showed significantly faster reaction times for error commission trials $v$. correct response trials $(t=7.11$, d.f. $=39, P<0.001)$ in keeping with the literature; ${ }^{32,33}$ and significant slowing across-group in reaction times for correct response trials following error commission $(t=6.37$, d.f. $=39$, $P<0.001)$, again in keeping with the literature. ${ }^{34}$ There were no significant between-group (controls $v$. MDD group) behavioural differences.

\section{Main task-based functional connectivity analysis (whole-brain level)}

\section{Across-group $(n=40)$ findings}

As expected the across-group $(n=40)$ positive and negative functional connectivity patterns were very similar for the two DMN seed regions in the right and left precuneus (illustrated using results from the right precuneus seed in Fig. 1). Thresholded at $t=5.23$, with clusters surviving $\mathrm{P}_{\mathrm{FDR} \text {-cor }}<0.05$ (and voxels surviving voxel-level $\left.\mathrm{P}_{\text {FWE-cor }}<0.05\right)$, regions of significant positive functional connectivity included bilateral precuneus and posterior cingulate cortex (Brodmann area (BA) 31); bilateral anterior cingulate cortex (BA 10, 32, 24); bilateral medial prefrontal cortex (BA 10); bilateral temporal cortex (BA 21); and bilateral posterior parietal cortex (BA 40). Regions of significant negative functional connectivity included bilateral supplementary motor area (BA 6); bilateral inferior orbitofrontal cortex (BA 11); bilateral anterior insula; bilateral precentral gyrus (BA 9); and bilateral dorsolateral prefrontal cortex (BA 10). These results are consistent with the literature. ${ }^{8}$

\section{Between-group findings}

At corrected whole-brain cluster-level $\mathrm{P}_{\mathrm{FDR} \text {-cor }}<0.05$ there was significantly greater functional connectivity for the MDD group $>$ controls from both right and left precuneus seeds to clusters in the right dorsomedial prefrontal cortex (BA 9) with some extension

Table 1 Demographic and clinical data for the major depressive disorder (MDD) and control groups

\begin{tabular}{|c|c|c|}
\hline & $\begin{array}{l}\text { Control group } \\
\qquad(n=20)\end{array}$ & $\begin{array}{l}\text { MDD group } \\
\qquad(n=20)\end{array}$ \\
\hline Age, years: mean (range) & $43(24-63)$ & $45(25-63)$ \\
\hline Male, $n(\%)$ & $6(30)$ & $7(35)$ \\
\hline Estimated IQ (from Quick), mean (range) & $108(98-130)$ & $104(90-120)$ \\
\hline Baseline Hamilton Rating Scale for Depression, mean (range) & $0.3(0-1)$ & $2(0-7)^{a}$ \\
\hline Beck Depression Inventory-II, mean (range) & $1.8(0-6)$ & $8.1(0-19)^{a}$ \\
\hline Neuroticism (EPQ-R), mean (range) & $2.9(0-8)$ & $8.9(3-12)^{a}$ \\
\hline First-degree relatives with MDD, $n$ (\%) & $1(5)$ & $14(70)^{\mathrm{a}}$ \\
\hline Age at first episode of MDD, years: mean (range) & N/A & $27(14-38)$ \\
\hline Episodes of MDD, mean (range) & N/A & $4(2-10)$ \\
\hline Time since remission, months: mean (range) & N/A & $13(3-36)$ \\
\hline Taking any antidepressant, $n(\%)$ & 0 & $14(70)^{\mathrm{a}}$ \\
\hline Group total of antidepressant medication (range) & 0 & $19(0-2)^{b}$ \\
\hline \multicolumn{3}{|c|}{$\begin{array}{l}\text { Quick, Ammons Quick Test; EPQ-R, Short Eysenck Personality Questionnaire - revised; N/A, not applicable. } \\
\text { a. Significant difference between the MDD and control groups, two-tailed } t \text {-tests } P<0.05 \text {. } \\
\text { b. Significant difference between the MDD and control groups and also within MDD group, with significantly greater amounts of antidepressant medication in the non-recurrence } \\
\text { group (two-tailed } t \text {-tests } P<0.05 \text { ). }\end{array}$} \\
\hline
\end{tabular}




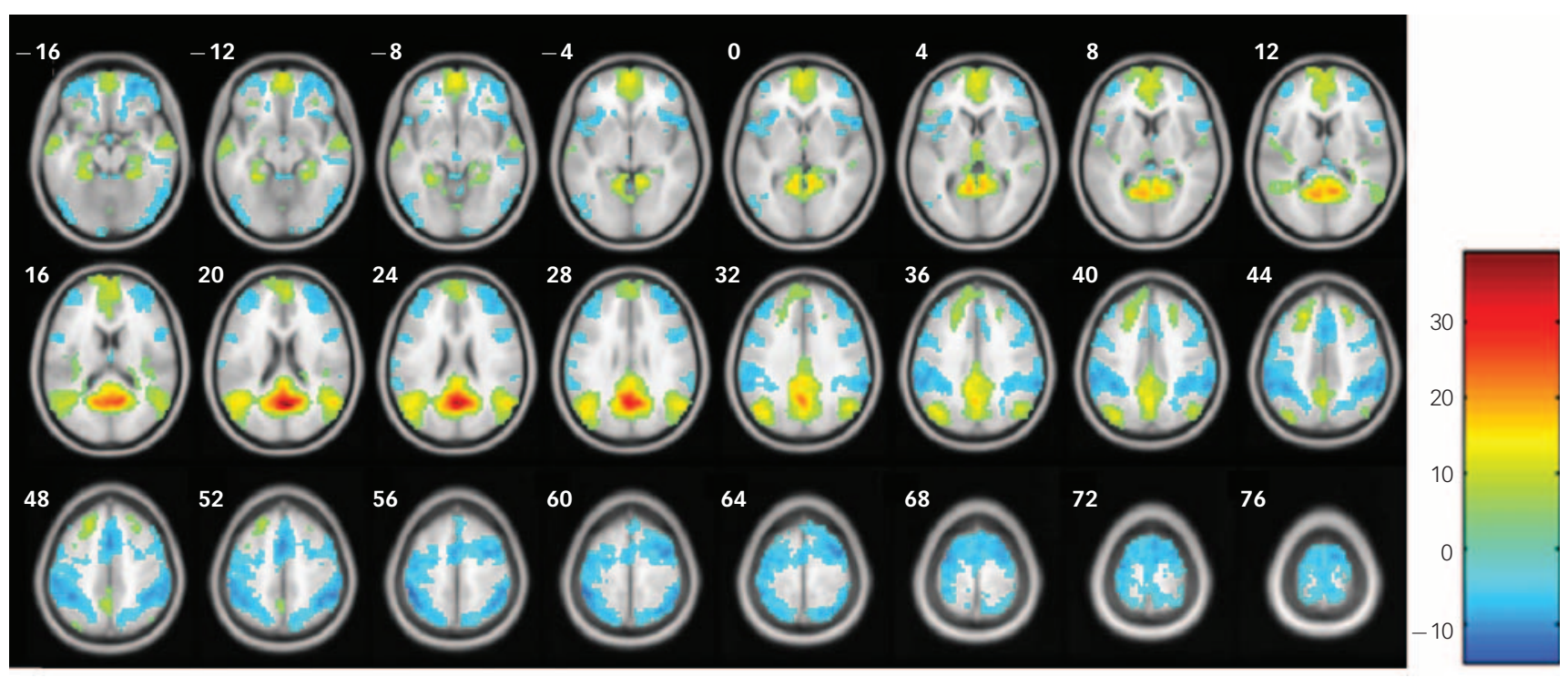

Fig. 1 Across-group $(n=40)$, whole-brain significant clusters.

Transverse slice view (on a $152 \mathrm{~T} 1$ xjview template) of significant positive functional connectivity thresholded at $t=5.23$ (red, yellow, green) and significant negative functional connectivity thresholded at $t=-5.23$ (blue) with the right precuneus (default mode network) seed $(7,-60,21)$. The colour bar indicates $t$ scores; figures adjacent to transverse slices are spatial $z$ coordinates.

into dorsal regions of the right frontal pole (BA 10) (statistics given in Table 2). There was also some evidence of increased functional connectivity (MDD group $>$ controls) to clusters in the left dorsomedial prefrontal cortex (BA 9), when thresholded at $P<0.001$, but this did not survive subsequent whole-brain correction (strongest from the right precuneus seed for the MDD group $>$ controls, cluster extent 20 voxels, cluster-level $\mathrm{P}_{\text {FDR-corr }}=0.054$ with peak voxel $t=4.85$ at $\left.-24,34,43\right)$. These findings are illustrated in Fig. 2. There were no cluster-level significant findings for the control>MDD group.

\section{Subsidiary analysis of medication status} (whole-brain level task-based functional connectivity) Three-group ANOVAs using medicated $(n=14)$, unmedicated $(n=6)$ and control $(n=20)$ groups investigated the effect of continuing maintenance-phase antidepressant medication (detailed in online Table DS1) on functional connectivity. The ANOVA of left precuneus seed functional connectivity showed significant main effect of group within a right dorsomedial prefrontal cortex cluster (peak at 30, 36, 39, $F_{2-75}=17.44, \mathrm{P}_{\mathrm{FWE}-\text { corr }}=0.042$ ). Follow-up $t$-tests, inclusively masked for a main effect of group at $P<0.001$, were significant for unmedicated patients $>$ controls at whole-brain level (cluster extent 28 voxels, cluster-level $\mathrm{P}_{\mathrm{FDR} \text {-corr }}=0.021$, peak $t=5.85$ at $30,36,39$ ). The ANOVA of right precuneus seed functional connectivity showed main effect that approached significance in the same right dorsomedial prefrontal cortex region (peak at $30,36,39, F_{2-75}=16.60, \mathrm{P}_{\mathrm{FWE}-\mathrm{corr}}=0.075$ ) and given the importance of exploring potential bias through medication follow-up $t$-tests were performed, masked for main effect of group at $P<0.001$, showing whole-brain significance for unmedicated patients $>$ controls (cluster 33 voxels, clusterlevel $\mathrm{P}_{\mathrm{FDR} \text {-corr }}=0.006$, peak $t=5.76$ at $\left.30,36,39\right)$. Figure 3 depicts these main between-group findings, using extracted data from $12 \mathrm{~mm}$ radius spheres centred on the peak voxel for main effect within the 3-group ANOVAs.

Prospective follow-up of the MDD group showed that positive medication status was associated with significantly reduced 1-year relapse (relative risk $=0.29,95 \%$ CI $0.10-0.85$ ).

\section{ROI task-based functional connectivity analysis}

A separate ROI analysis assessed the proximity of our whole-brain dorsomedial prefrontal cortex findings to specific bilateral dorsomedial prefrontal cortex 'dorsal nexus' regions from the literature (left ROI $-24,35,28$; right ROI 18, 34, 29). ${ }^{11}$ Results of this ROI analysis are presented in Table 3 , showing significantly increased task-based functional connectivity, surviving clusterlevel correction $\mathrm{P}_{\mathrm{FDR}-\text { cor }}<0.05$, between bilateral precuneus regions and clusters within a $12 \mathrm{~mm}$ radius sphere centred on the bilateral dorsomedial prefrontal cortex 'dorsal nexus' regions.

\section{Gyrification analysis}

There was significant bilateral hypogyrification (cluster-wise $P<0.001$, permutation corrected for multiple testing, cluster inclusion threshold $P=0.05, n=10000$ ) in the MDD group extending across bilateral medial surface regions incorporating the precuneus. Additionally, there was hypergyrification (clusterwise $P<0.001$, permutation corrected for multiple testing, cluster inclusion threshold $P=0.05, n=10000$ ) in the MDD group in a more anterior region incorporating the left anterior cingulate cortex. These results are illustrated in Fig. 4.

To assess the effect of medication in this gyrification analysis we compared medicated and unmedicated patients at the lenient statistical threshold of $P=0.10$ but did not detect any significant differences. The results of this analysis were unaffected when re-analysed using ICV as an additional covariate.

As shown in Fig. 4, the left precuneus hypogyrification incorporated the precuneus (DMN) seed used in our functional connectivity analysis and originally identified by Sheline et al. ${ }^{11}$ Additionally, we mapped Sheline et al's left 'dorsal nexus' ( -24 , 35,28 spherical ROI of $12 \mathrm{~mm}$ radius) onto the inflated surface to study the overlap with the gyrification maps. The BA 32 part of 'dorsal nexus' (but not the BA 8 or BA 9 areas) showed an overlap with the hypergyric anterior cingulate cortex cluster identified here. Task-based functional connectivity analysis based on this hypergyric anterior cingulate cortex cluster (presented in the online data supplement) identified only one significant finding, of hyperconnectivity for the control $>$ MDD group within 


\begin{tabular}{|c|c|c|c|c|c|c|}
\hline MDD group > control group & $\begin{array}{l}\text { Cluster size } \\
\text { voxels }\end{array}$ & $\begin{array}{c}\text { Region } \\
\text { Brodmann area }\end{array}$ & Cluster-level $P_{\text {FDR-cor }}$ & Peak $t$ score & Peak Z score & $\begin{array}{c}\text { Peak coordinates } \\
x, y, z\end{array}$ \\
\hline \multicolumn{7}{|l|}{ Right DMN seed } \\
\hline Right dmPFC & 45 & 9 & 0.001 & 4.45 & 4.18 & $30,40,34$ \\
\hline \multicolumn{7}{|l|}{ Left DMN seed } \\
\hline Right dmPFC & 27 & 9 & 0.025 & 4.46 & 4.19 & $30,40,34$ \\
\hline Right dmPFC & 25 & 10 & 0.025 & 4.57 & 4.28 & $21,56,14$ \\
\hline
\end{tabular}
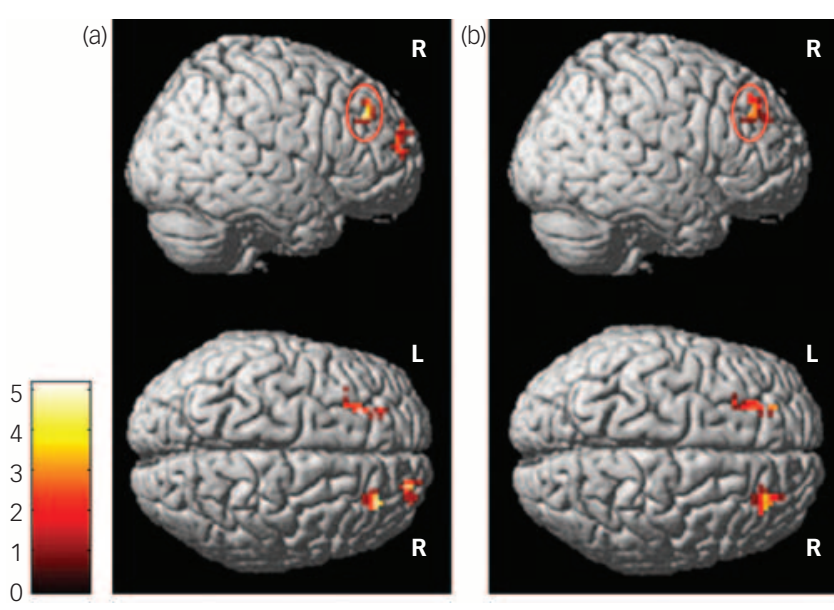

Fig. 2 Rendered images (xjview) of functional connectivity from the (a) left and (b) right precuneus (default mode network (DMN)) seeds for the major depressive disorder group $>$ control group.

Thresholded at $P<0.001$ uncorrected, cluster size $>5$ voxels. All right cortical clusters shown here survived subsequent correction at cluster-level false discovery ratecorrected $P<0.05$; left cortical clusters did not survive correction at this level. The superimposed red outline on the right lateral view indicates the most consistent finding of whole-brain significant hyperconnectivity from both left and right precuneus (DMN) seeds to an overlapping area of dorsomedial prefrontal cortex (BA 9), also significant seeds to an overlapping area of dorsomedial prefrontal cortex (BA 9), also significant
within a small volume correction of the right 'dorsal nexus' described by Sheline within a small volume correction of the right 'dorsal nexus' described by Sheline
et al. ${ }^{8}$ The red outline on the upper images indicates clusters with a 24 -voxel overlap et $a l^{8}$ The red outline on the upper images indicates clusters with a 24-voxel over
(peak $30,40,34$ ). Upper images are in lateral view and lower images in axial view. (peak 30, 40, 34). Upper images are in lateral view and lower images in axial view.
The colour bar indicates $t$ scores.

a left posterior temperoparietal area (cluster 33 voxels, clusterlevel $\mathrm{P}_{\mathrm{FDR} \text {-corr }}=0.005$, peak $t=4.24$ at $\left.-45,-48,27\right)$.

\section{Discussion}

Our data provide the first direct evidence of task-based DMN hyperconnectivity within recovered-state MDD, confirming a recently published hypothesis. ${ }^{10}$ This adds to resting-state functional connectivity evidence from depressed-state $\mathrm{MDD}^{11}$ and neverdepressed, high vulnerability cohorts, ${ }^{9}$ supporting the idea that DMN hyperconnectivity is a core feature of the highly recurrent disorder of MDD, extending across different states into apparent clinical recovery. Viewed as a biological substrate, the persistence of DMN hyperconnectivity into recovery is consistent with wellestablished psychological models that have long identified the persistence of psychological-level risk factors into recovered-state MDD. ${ }^{34}$ More specifically, because of its association with internal focus, DMN hyperconnectivity is a plausible substrate for rumination, observed across states of MDD and described by Beck as involving disproportionate allocation of resources from, 'the external environment to internal experiences., ${ }^{35}$ At a brain network level, 'the interference hypothesis' holds that persistence

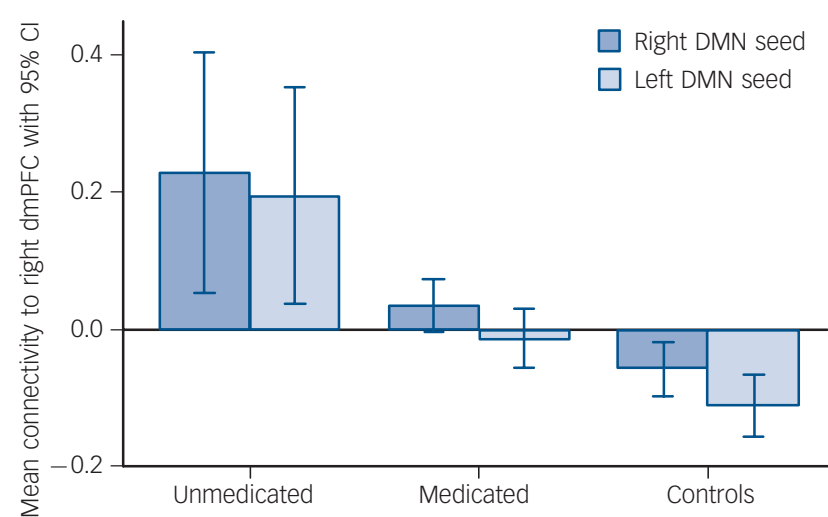

Fig. 3 Functional connectivity between precuneus (default mode network, DMN) seeds (black for right, grey for left) and the right dorsomedial prefrontal cortex (dmPFC, 30, 36, 39), for unmedicated, medicated and control groups.

Extracted data from $12 \mathrm{~mm}$ radius spheres centred on the peak voxel for main effect within the three-group ANOVA (with error bars indicating $95 \%$ confidence intervals).

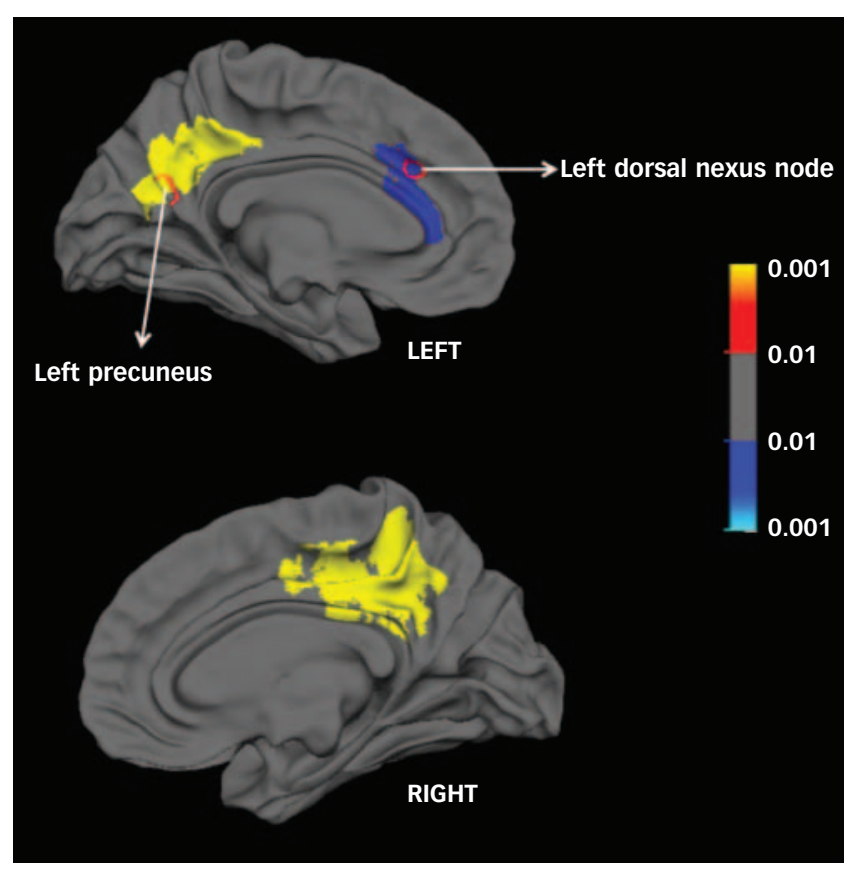

Fig. 4 Depiction of whole-brain significant findings from the gyrification analysis.

Shows significant $(P<0.001)$ bilateral precuneus hypogyrification (yellow); and hypergyrification (blue) within the left dorsal and rostral anterior cingulate cortex for the major depressive disorder group compared with the controls. The colour bar represents $P$-values. The circular regions of interest (indicated in red outline) depict the left precuneus default mode network seed used in our functional connectivity analysis (taken from Sheline et $a^{8}$ ) and a part of Sheline et al's 'dorsal nexus'. 
Table 3 Results of the region of interest analysis based on coordinates for the dorsomedial prefrontal cortex 'dorsal nexus' regions given in Sheline et $\left.a\right|^{8, a}$

\begin{tabular}{|c|c|c|c|c|c|c|}
\hline MDD group > control group & $\begin{array}{l}\text { Cluster size } \\
\text { voxels }\end{array}$ & $\begin{array}{c}\text { Region } \\
\text { Brodmann area }\end{array}$ & Cluster-level $P_{\text {FDR-cor }}$ & Peak $t$ score & Peak z score & $\begin{array}{c}\text { Peak coordinates } \\
x, y, z\end{array}$ \\
\hline \multicolumn{7}{|l|}{ Right precuneus } \\
\hline Right dmPFC & 20 & 9 & 0.002 & $4.10^{*}$ & 3.88 & $24,39,28$ \\
\hline \multicolumn{7}{|l|}{ Left precuneus } \\
\hline Right dmPFC & 10 & 9 & 0.040 & $4.04^{*}$ & 3.83 & $27,36,29$ \\
\hline Left dmPFC & 15 & 9 & 0.006 & $3.70 *$ & 3.54 & $-21,31,29$ \\
\hline
\end{tabular}

of relatively high DMN connectivity into task interferes with adaptive switching to more appropriate goal-oriented brain networks. ${ }^{36}$ More recently there have been indications that these networks overlap to an unusual degree within MDD (reviewed in Marchetti et $a l^{10}$ ) and that the most robustly identified hub in our task-based DMN analysis (right dorsomedial prefrontal cortex, BA 9) acts as a 'dorsal nexus', strongly connected to several networks within the depressed-state of MDD. ${ }^{11}$ Since we have previously shown event-related fMRI hypoactivity within this right 'dorsal nexus' region during active processing (for example of error commission) in recovered-state $\mathrm{MDD},{ }^{5}$ then invoking the 'interference hypothesis ${ }^{36}$ supports the proposition that within recovered-state MDD persistent excessive DMN activity during task, most prominently involving the right 'dorsal nexus', interferes with capacity to appropriately switch this dorsomedial prefrontal cortex (BA 9) region to its role in more 'task positive' (active) networks; resulting in cognitive and attentional bias that increases the risk of depression.

The functional connectivity findings were associated with bilateral hypogyrification incorporating the precuneus, broadly consistent with the only previous MDD gyrification study that we are aware of, which did not incorporate a functional connectivity analysis but demonstrated hypogyrification in a depressed-state MDD cohort extending bilaterally into posterior cingulate/precuneus areas. ${ }^{37}$ Our data extend this finding by identifying a link between hypogyrification of key DMN hubs (bilateral precuneus) and task-based DMN hyperconnectivity in the recovered MDD group (20 patients $v$. 20 controls). In attempting to understand the directionality of this association, we can draw on limited recent evidence from multimodal work in schizophrenia, showing a link between regional hypergyrification and reduced long-range functional connectivity, ${ }^{12}$ consistent with the converse finding here of hypogyrification associated with increased long-range functional connectivity (between the precuneus and dorsomedial prefrontal cortex in the MDD group); and also consistent with the functional connectivity analysis based on MDD group anterior cingulate cortex hypergyrification (associated with relatively reduced long-range functional connectivity in patients). Since the anterior cingulate cortex has been viewed in the literature as part of a salience network, ${ }^{38}$ distinct from the DMN, the increased functional connectivity in controls between the left anterior cingulate cortex and left temperoparietal regions involved in external cue processing (including speech) could potentially have a functional advantage through enhanced environmental awareness.

Limited evidence from our subsidiary analyses showed no apparent effect of medication status on DMN node gyrification (i.e. significant precuneus hypogyrification was only observed when comparing the whole MDD group against controls); but a significant association between medication status and task-based functional connectivity, consistent for bilateral precuneus seeds and producing an apparently stratified effect (shown in Fig. 3). Although we must remain cautious, since one of the groups was small $(n=6)$, the results do at least raise the possibility of a relatively stable structural disruption involving key DMN nodes (observable in significant hypogyrification for the whole MDD group $v$. control group, without apparent medication status effects), linked to relatively modifiable task-based DMN hyperconnectivity (associated with significant medication status effects). In this case DMN gyrification and connectivity patterns might be thought of as different biological levels of MDD vulnerability, with the former potentially related to early disruptive effects of trauma on structural aspects of DMN development ${ }^{8}$ within the main period of gyrus formation. ${ }^{39}$

\section{Limitations}

We acknowledge the potential bias introduced by maintenancephase antidepressant medication in the MDD group. However, additional targeted analyses did not find any association between either task-based functional connectivity or gyrification and positive medication status. Indeed, where there was any association with medication status (in task-based functional connectivity), this was most abnormal (from both DMN seeds) in patients who had stopped maintenance-phase medication prior to scanning. Although we could not find any indication that maintenance-phase antidepressant medication caused the observed differences in DMN functional connectivity or gyrification, we should remain cautious that this part of the analysis (relying on relatively small groups) may have been underpowered to detect real differences.

Additionally, we have presented a seed-region DMN functional connectivity analysis using task-based data, following on from extensive literature indicating the validity of this approach (for example Rissman et al, ${ }^{23}$ Hampson et al, ${ }^{24}$ Fair et $a l^{40}$ ), and evidence of persisting, although relatively attenuated DMN activation during a range of tasks, including externally focused activity. ${ }^{40,41}$ Although this approach has been shown to provide qualitatively similar results to the analysis of resting-state functional connectivity data, caution should be exercised in the quantitative differences that may exist between these approaches. ${ }^{40}$ This issue is likely to be most problematic when making withingroup task-based $v$. resting-state functional connectivity comparisons and should be much less problematic in the analysis presented here, where we made between-group comparisons focused purely on task-based DMN functional connectivity (i.e. not making experimental comparisons with resting-state). We should also remain conscious of this issue in considering resting-state and task-based studies from the literature; different approaches that often retain mutual relevance, as here.,11 


\section{Strengths}

We present the first data on DMN connectivity (task-based) in recovered-state MDD and provide the first multimodal analysis relating functional connectivity findings in MDD to cortical gyrification. These data test a specific and important hypothesis coming out of the MDD literature.

\section{Implications}

Within apparent clinical recovery from MDD there are significant and apparently linked abnormalities in DMN structure (identified through hypogyrification of key hubs) and function (identified through task-based DMN hyperconnectivity). These new findings strengthen our knowledge of the biological-level vulnerability to MDD by confirming and extending an important hypothesis ${ }^{10}$ based on previous research. ${ }^{9,11}$ When assessed alongside eventrelated fMRI evidence, ${ }^{5}$ it seems possible that the identified task-based DMN hyperconnectivity may interfere with switching of important dorsomedial prefrontal cortex areas to more appropriate task-positive activity, in line with earlier theories. ${ }^{36}$ Further research is needed to confirm the more limited findings that DMN functional hyperconnectivity is greatest in patients with MDD who have discontinued maintenance-phase medication; and the theory that this networked brain activity may represent a more dynamic indicator of MDD vulnerability linked to relatively stable cortical gyrification abnormalities.

N. L. Nixon, MBBS, MRCPsych, MMedSci, P. F. Liddle, BMBCh, MRCPsych, PhD, E. Nixon, PhD, G. Worwood, MBBCh, MRCPsych, MMedSci, Division of Psychiatry E. Nixon, PhD, G. Worwood, MBBCh, MRCPsych, MMedSci, Division of Psychict
and Applied Psychology, Institute of Mental Health, University of Nottingham, Nottingham, UK; M. Liotti, MD, PhD, Department of Psychology, Simon Fraser University, Burnaby, Canada; L. Palaniyappan, MBBS, MRCPsych, PhD, Centre for Translational Neuroimaging, Division of Psychiatry and Applied Psychology, Institute of Mental Health, University of Nottingham, Nottingham, UK

Correspondence: N. L. Nixon, Division of Psychiatry, Institute of Mental Health, University of Nottingham, Triumph Road, Nottingham NG7 2TU, UK. Email neil.nixon@nottingham.ac.uk

First received 3 Apr 2013, final revision 12 Aug 2013, accepted 25 Oct 2013

\section{References}

1 Judd LL. The clinical course of unipolar major depressive disorders. Arch Gen Psychiatry 1997; 54: 989-91.

2 Waraich P, Goldner EM, Somers JM, Hsu L. Prevalence and incidence studies of mood disorders: a systematic review of the literature. Can J Psychiatry 2004; 49: 124-38.

3 Beck AT. Cognitive models of depression. J Cogn Psychother 1987; 1: 5-37.

4 Abramson LY, Metalsky GI, Alloy LB. Hopeless depression: a theory-based subtype of depression. Psychol Rev 1989; 96: 358-72.

5 Nixon NL, Liddle PF, Worwood G, Liotti M, Nixon E. Prefrontal cortex function in remitted major depressive disorder. Psychol Med 2013; 43: 1219-30.

6 Norbury R, Selvaraj S, Taylor MJ, Harmer C, Cowen PJ. Increased neural response to fear in patients recovered from depression: a $3 \mathrm{~T}$ functional magnetic resonance imaging study. Psychol Med 2010; 40: 425-32.

7 Raichle M, MacLeod MA, Snyder AZ, Powers WJ, Gusnard DA, Shulman GL. A default mode of brain function. Proc Natl Acad Sci U S A 2001; 98: 676-82.

8 Sheline YI, Barch DM, Price JL, Rundle MM, Vaishnavi SN, Snyder AZ, et al. The default mode network and self-referential processes in depression. Proc Natl Acad Sci U S A 2009; 106: 1942-7.

9 Norbury R, Mannie Z, Cowen PJ. Imaging vulnerability for depression. Mol Psychiatry 2011; 16: 1067-8.

10 Marchetti I, Koster EHW, Sonuga-Barke EJ, De Raedt R. The default mode network and recurrent depression: a neurobiological model of cognitive risk factors. Neuropsychol Rev 2012; 22: 229-51.

11 Sheline YI, Price JL, Yan Z, Mintun MA. Resting-state functional MRI in depression unmasks increased connectivity between networks via the dorsal nexus. Proc Natl Acad Sci U S A 2010; 107: 11020-5.

12 Dauvermann MR, Mukherjee $\mathrm{P}$, Moorhead WT, Stanfield AC, Fusar-Poli $\mathrm{P}$, Lawrie SM, et al. Relationship between gyrification and functional connectivity of the prefrontal cortex in subjects at high genetic risk of schizophrenia. Curr Pharm Des 2012; 18: 434-42.

13 First MB, Spitzer RL, Gibbon M, Williams JBW. Structured Clinical Interview for DSM-IV Axis I Disorders - Clinician Version (SCID-CV). American Psychiatric Press, 1997

14 Hamilton M. A rating scale for depression. J Neurol Neurosurg Psychiatry 1960; 23: 56-62.

15 Beck AT, Steer RA, Ball R, Ranieri WF. Comparison of Beck Depression Inventories-IA and -II in psychiatric outpatients. J Pers Assess 1996; 67: 588-97.

16 Eysenck HJ, Eysenck SBG. Manual of the Eysenck Personality Scales. Hodder and Stoughton, 1991.

17 Hyler SE. Personality Diagnostic Questionnaire (PDQ-4+). New York State Psychiatric Institute, 1994.

18 Ammons RB, Ammons $\mathrm{CH}$. The Quick test: provisional manual. Psychol Rep 1962; 11: 111-61.

19 Folstein MF, Folstein SE, McHugh PR. 'Mini-mental state'. A practical method for grading the cognitive state of patients for the clinician. J Psychiatr Res 1975; 12: 189-98.

20 Oldfield RC. The assessment and analysis of handedness: the Edinburgh inventory. Neuropsychologia 1971; 9: 97-113.

21 American Psychiatric Association. Diagnostic and Statistical Manual of Mental Disorders (4th edn) (DSM-IV). APA, 1994.

22 Yan C, Zang Y. DPARSF: a MATLAB toolbox for "pipeline" data analysis of resting-state fMRI. Front Syst Neurosci 2010; 4: 13.

23 Rissman J, Gazzaley A, D'Esposito M. Measuring functional connectivity during distinct stages of a cognitive task. Neuroimage 2004; 23: 752-63.

24 Hampson M, Driesen N, Skudlarski P, Gore J, Constable RT. Brain connectivity related to working memory performance. J Neurosci 2006; 26: 13338-43.

25 Brett M, Christoff K, Cusack R, Lancaster J. Using the Talairach atlas with the MNI template. Neuroimage 2001; 16: S85.

26 Chumbley JR, Friston KJ. False discovery rate revisited: FDR and topological inference using Gaussian random fields. Neuroimage 2009; 44: $62-70$.

27 Dale AM, Fischl B, Sereno MI. Cortical surface-based analysis: I. Segmentation and surface reconstruction. Neuroimage 1999; 9 : 179-94.

28 Schaer M, Cuadra MB, Tamarit L, Lazeyras F, Eliez S, Thiran J-P. A surface-based approach to quantify local cortical gyrification. Trans Med Imagaging 2008; 27: 161-70.

29 Zilles K, Armstrong E, Schleicher A, Kretschmann H-J. The human pattern of gyrification in the cerebral cortex. Anat Embryol 1988; 179: 173-9.

30 Palaniyappan L, Liddle PF. Aberrant cortical gyrification in schizophrenia: a surface-based morphometry study. J Psychiatry Neurosci 2012; 37: 17-27.

31 Fan Q, Palaniyappan L, Tan L, Wang J, Wang X, Li C, et al. Surface anatomical profile of the cerebral cortex in obsessive-compulsive disorder: a study of cortical thickness, folding and surface area. Psychol Med 2013; 43: 1081-91.

32 Ruchsow M, Herrnberger B, Beschoner $\mathrm{P}$, Groon G, Spitzer M, Keifer M. Error processing in major depressive disorder: evidence from event-related potentials. J Psychiatr Res 2006; 40: 37-46.

33 Holmes AJ, Pizzagalli DA. Spatio-temporal dynamics of error processing dysfunctions in major depressive disorder. Arch Gen Psychiatry 2008; 65: $179-88$

34 Laming DRJ. Information Theory of Choice-Reaction Times. Academic Press, 1968.

35 Beck AT. The evolution of the cognitive model of depression and its neurobiological correlates. Am J Psychiatry 2008; 165: 969-77.

36 Sonuga-Barke EJ, Castellanos F. Spontaneous attentional fluctuations in impaired states and pathological conditions: a neurobiological hypothesis. Neurosci Biobehav Rev 2007; 31: 977-86.

37 Zhang Y, Yu C, Zhou Y, Li K, Li C, Jiang T. Decreased gyrification in major depressive disorder. Neuroreport 2009; 20: 378-80.

38 Sridharan $D$, Levitin DJ, Menon V. A critical role for the right fronto-insular cortex in switching between central-executive and default-mode networks. Proc Natl Acad Sci U S A 2008; 105: 12569-74.

39 White T, Hilgetag CC. Gyrification and neural connectivity in schizophrenia Dev Psychopathol 2011; 23: 339-52.

40 Fair D, Schlaggar B, Cohen A, Miezin F, Dosenbach N, Wenger K, et al. A method for using blocked and event-related fMRI data to study "resting state" functional connectivity. Neuroimage 2007; 35: 396-405

41 Duan X, Liao W, Liang D, Lihua Q, Qing G, Liu C, et al. Large-scale brain networks in board game experts: insights from a domain-related task and task-free resting state. PLOS One 2012; 7: 1-10. 